\title{
Neutrophil-to-lymphocyte ratio predicts overall survival of patients with combined hepatocellular cholangiocarcinoma
}

\author{
CHAOBIN HE*, YIZE MAO*, XIANGMING LAO, SHENGPING LI and XIAOJUN LIN \\ Department of Hepatobiliary Oncology, Cancer Center, State Key Laboratory of Oncology in South China, \\ Collaborative Innovation Center for Cancer Medicine, Sun Yat-sen University, Guangzhou, Guangdong 510060, P.R. China
}

Received May 5, 2016; Accepted July 20, 2017

DOI: $10.3892 / \mathrm{ol} .2018 .7882$

\begin{abstract}
The neutrophil-to-lymphocyte ratio (NLR) has been regarded as a prognostic factor in various types of cancer. The present study aimed to identify the association between NLR and combined hepatocellular cholangiocarcinoma (cHCC-CC) in patients who underwent surgical resection. The present study retrospectively reviewed 59 patients who were diagnosed with $\mathrm{cHCC}-\mathrm{CC}$ and treated with surgical resection between January 2000 and October 2014 at the Department of Hepatobiliary and Pancreatic Surgery at Sun Yat-sen University Cancer Center (Guangzhou, China). The patients were divided into two groups: NLR $\leq 2.75$ and NLR $>2.75$. Patients with stage I and II or stage III and IV disease were classified into early- and advanced-stage groups, respectively, according to the Tumor-Node-Metastasis (TNM) staging system. Overall survival time (OS) was estimated using the Kaplan-Meier method. Univariate and multivariate Cox regression models were used to evaluate the prognostic value of NLR. The NLR value was significantly higher in the HCC advanced-stage group compared with that in the HCC early-stage group according to the TNM staging system (3.19 vs. $2.00 ; \mathrm{P}=0.001)$. The median survival time was 83.6 months in the NLR $\leq 2.75$ group and 15 months in the NLR $>2.75$ group $(\mathrm{P}=0.004)$. Upon multivariate analysis, NLR $>2.75$ was an independent prognostic factor for poor cHCC-CC outcomes. Overall, the easily evaluated pre-treatment NLR may be an independent prognostic factor for patients with $\mathrm{cHCC}-\mathrm{CC}$ treated by surgical resection.
\end{abstract}

Correspondence to: $\mathrm{Dr}$ Shengping $\mathrm{Li}$ or $\mathrm{Dr}$ Xiaojun Lin, Department of Hepatobiliary Oncology, Cancer Center, State Key Laboratory of Oncology in South China, Collaborative Innovation Center for Cancer Medicine, Sun Yat-sen University, 651 Dongfeng Road East, Guangzhou, Guangdong 510060, P.R. China

E-mail: lishp@sysucc.org.cn

E-mail:linxj@sysucc.org.cn

*Contributed equally

Key words: combined hepatocellular cholangiocarcinoma, neutrophil-to-lymphocyte ratio, predict, overall survival, prognosis

\section{Introduction}

Combined hepatocellular cholangiocarcinoma (cHCC-CC), a rare and unique form of primary liver malignancy, was first described in 1949 by Allen and Lisa (1), and accounts for $0.4-14.2 \%$ of primary liver malignancies $(2,3)$. The World Health Organization classification defines cHCC-CC as a tumor containing unequivocal $\mathrm{HCC}$ and $\mathrm{CC}$ components; the condition is distinguished from separate $\mathrm{HCC}$ and intrahepatic CC (ICC) arising in the same liver (4).

The prognosis of patients with $\mathrm{cHCC}-\mathrm{CC}$ undergoing liver resection has been reported to be poor. The 5-year overall survival (OS) rate was reported to be $37.2 \%$, and the 5-year disease free survival rate was $10.7 \%$ (5); therefore, it is necessary to identify the prognostic factors of patients diagnosed with cHCC-CC who underwent surgical resection.

Due to its low prevalence, the prognostic factors of $\mathrm{cHCC}-\mathrm{CC}$ remain unclear. The well-known Tumor-Node-Metastasis (TNM) staging system for $\mathrm{CHCC}-\mathrm{CC}$ remains controversial and poorly understood (6). Whether common prognostic factors (particularly lymph nodal metastasis, which has been most frequently revealed to be associated with the prognosis of various solid tumors, including HCC and ICC) $(5,7)$ leads to a poorer prognosis in patients with $\mathrm{HCC}-\mathrm{CC}$ is also controversial (8). Certain previous studies demonstrated that lymph node metastasis was a significant prognostic factor in patients with cHCC-CC (5), while in other reports (6), lymphatic metastasis failed to represent a significant prognostic factor. Thus, novel prognostic markers are required to predict the prognosis of patients with $\mathrm{cHCC}-\mathrm{CC}$.

Inflammation serves an important role in the development and progression of numerous malignancies by participating in the neoplastic process, proliferation and migration (9). Systemic inflammation is a complex progress, the response to which can be assessed using the neutrophil-to-lymphocyte ratio (NLR) (10). Elevated pre-treatment NLR has been confirmed to be associated with poor outcomes in various types of cancer, including non-small cell lung cancer (11), gastric cancer (12), ovarian cancer (13), advanced pancreatic cancer (14), hepatocellular carcinoma (15-19) and cholangiocarcinoma (20). However, the utility of the NLR has not been validated in $\mathrm{cHCC}-\mathrm{CC}$.

The present study hypothesized that the NLR may be a practical predictor of the inflammatory process, and 
investigated the association between inflammation and the prognosis of patients with $\mathrm{cHCC}-\mathrm{CC}$. Therefore, the present retrospective study evaluated the association between the NLR and prognosis in patients with $\mathrm{cHCC}-\mathrm{CC}$ who underwent surgical resection.

\section{Patients and methods}

Patient cohort. A total of 59 patients who underwent surgical resection and were histologically diagnosed with $\mathrm{cHCC}-\mathrm{CC}$ between January 2000 and October 2014 at the Department of Hepatobiliary and Pancreatic Surgery (Sun Yat-sen University Cancer Center, Guangzhou, China) were retrospectively recruited. Exclusion criteria were as follows: i) other treatments, including transarterial chemoembolization, radiofrequency treatment and liver transplantation for patients with HCC before surgical resection; ii) inadequate renal function (serum creatinine level and blood urea nitrogen level higher than the upper limits of normal); iii) severe coagulopathy (prothrombin activity $<40 \%$ or platelet count $<40,000 / \mathrm{mm}^{3}$ ); iv) Child-Pugh $C$ liver function or evidence of hepatocellular decompensation, including refractory ascites, esophageal or gastric variceal bleeding, and hepatic encephalopathy; v) obstructive jaundice; vi) other concurrent primary tumors; vii) pathological confirmed subtype of $\mathrm{cHCC}-\mathrm{CC}$ with stem cell features or viii) follow-up period of $<3$ months or lost to follow up.

Clinical data collection. All clinicopathological data were retrieved from medical records at the Department of Hepatobiliary and Pancreatic Surgery of the Sun Yat-sen University Cancer Center. Clinicopathological parameters included histologically confirmed cHCC-CC, age, gender, leukocyte cell count, neutrophil cell count and lymphocyte cell count, levels of hemoglobin, platelets, $\alpha$-fetoprotein (AFP), carbohydrate antigen 19-9 (CA19-9), $\gamma$-glutamyl transpeptidase (GGT) and hepatitis B surface antigen (HbsAg), tumor size, tumor number, lymph node metastasis, major thrombi, microvascular thrombi, and conventional TNM stage for HCC and ICC, as established by the Union for International Cancer Control and the American Joint Committee on Cancer (AJCC) (21) (Table I). The laboratory data were obtained prior to surgical resection. The present study was approved by the Institutional Review Board of the Sun Yat-sen University Cancer Center. Written informed consent was obtained from all patients prior to enrollment in the present study.

Follow-up. Patients were followed up at least every 2 months during the first year and every 3 months thereafter. Tumor markers, including AFP, CEA and CA19-9 tests, liver ultrasonography, computed tomography and magnetic resonance imaging, were selected as required. OS was defined as the duration (in months) from the date of surgery until cancer-specific mortality or last follow-up. The final follow-up date was August 1, 2015.

Statistical analysis. The optimal cut-off values for NLR were determined using time-dependent receiver operating curve (ROC) analysis. Time-dependent ROC analysis was performed using $\mathrm{R}$ software version 3.2.2 (The $\mathrm{R}$ Foundation for
Statistical Computing, Vienna, Austria; http://www.r-project. org) and the 'survival ROC' package (22). The NLR was evaluated by dividing the neutrophil cell counts by the lymphocyte cell counts. The NLR value was categorized into two groups: NLR $\leq 2.75$ and NLR $>2.75$.

SPSS version 22 (IBM Corp., Armonk, NY, USA) was used to analyze the data. Continuous variables were expressed as the mean \pm standard deviation and the range, and were compared between the NLR $\leq 2.75$ and NLR $>2.75$ groups using Student's t-test. The $\chi^{2}$ test and Fisher's exact test were used to compare categorical variables, which were presented as the number and percentage of patients.

Survival curves for OS were analyzed using the Kaplan-Meier method. Significant differences between groups were identified using the log-rank test. A univariate analysis was performed to assess significant differences in clinicopathological characteristics. A multivariate analysis was performed via Cox regression for variables significant in a univariate analysis, and the associated $95 \%$ confidence interval (CI) was determined. $\mathrm{P}<0.05$ was considered to indicate a statistically significant difference.

\section{Results}

Patient characteristics. A total of 59 patients were diagnosed with cHCC-CC between January 2000 and October 2014, and consecutively enrolled in the present retrospective study. The median patient age was 49 years (range, 25-75 years). Among these patients, $43(72.9 \%)$ were male and $16(27.1 \%)$ were female. There were $40(67.8 \%)$ patients in the HCC early-stage group and 19 (32.2\%) patients in the HCC advanced-stage group, according to the TNM staging system. Furthermore, according to the ICC TNM staging system, 45 (76.3\%) patients were in the early-stage group and $14(23.7 \%)$ patients were in the advanced-stage group at diagnosis.

Among the patients diagnosed with $\mathrm{cHCC}-\mathrm{CC}, 52$ were included in the present study for prognostic analysis; the remaining 7 were excluded, as their survival time was $<3$ months. The study cohort for the prognostic analysis consisted of 37 (71.2\%) males and 15 (28.8\%) females, and was prospectively recruited and retrospectively analyzed. The median age of the patients in the prognostic analysis was 50 years (range $27-75$ years).

The clinicopathological characteristics of the investigated patients in the prognostic analysis are presented in Table I. A total of 8 patients $(15.4 \%)$ were older than 60 years, and the majority of the patients $(71.2 \%)$ were male. Depending on the selected NLR value, patients were divided into two groups: NLR $\leq 2.75$ and NLR $>2.75$. A total of 41 patients $(78.8 \%)$ were in the NLR $\leq 2.75$ group, whereas 11 patients $(21.2 \%)$ were in the NLR>2.75 group. There were no significant differences regarding age, gender, AFP, CA19-9, GGT, HbsAg, tumor number, lymph node metastasis, major thrombi, microvascular thrombi, HCC stage or ICC stage between the two groups; however, tumor size was significantly larger in the NLR $>2.75$ group compared with that in the NLR $\leq 2.75$ group (log-rank test, $\mathrm{P}=0.017$ ).

Association between NLR and tumor stage. The association between NLR and tumor stage, which was the primary 
Table I. Clinicopathological factors in NLR $\leq 2.75(n=41)$ and NLR $>2.75(n=11)$ groups at diagnosis.

\begin{tabular}{|c|c|c|c|}
\hline Variables & $\begin{array}{c}\text { NLR } \leq 2.75 \\
\mathrm{n}\end{array}$ & $\begin{array}{c}\text { NLR }>2.75 \\
\mathrm{n}\end{array}$ & P-value \\
\hline \multicolumn{4}{|l|}{ Age, years } \\
\hline$\leq 60$ & 35 & 9 & \\
\hline$>60$ & 6 & 2 & 0.856 \\
\hline \multicolumn{4}{|l|}{ Gender } \\
\hline Female & 11 & 4 & \\
\hline Male & 30 & 7 & 0.709 \\
\hline \multicolumn{4}{|l|}{$\mathrm{AFP}, \mathrm{ng} / \mathrm{ml}$} \\
\hline$\leq 25$ & 16 & 4 & \\
\hline$>25$ & 25 & 7 & 0.851 \\
\hline \multicolumn{4}{|l|}{ CA19-9, U/ml } \\
\hline$\leq 35$ & 27 & 9 & \\
\hline$>35$ & 14 & 2 & 0.468 \\
\hline \multicolumn{4}{|l|}{ GGT, U/1 } \\
\hline$\leq 50$ & 21 & 2 & \\
\hline$>50$ & 20 & 9 & 0.086 \\
\hline \multicolumn{4}{|l|}{ HbsAg } \\
\hline Negative & 5 & 1 & \\
\hline Positive & 36 & 10 & 0.806 \\
\hline \multicolumn{4}{|l|}{ Tumor size, cm } \\
\hline$<5$ & 24 & 2 & \\
\hline$\geq 5$ & 17 & 9 & 0.017 \\
\hline \multicolumn{4}{|l|}{ Tumor number } \\
\hline Solitary & 11 & 3 & \\
\hline Multiple & 30 & 8 & 0.724 \\
\hline \multicolumn{4}{|c|}{ Lymph node metastasis } \\
\hline Negative & 37 & 8 & \\
\hline Positive & 4 & 3 & 0.154 \\
\hline \multicolumn{4}{|l|}{ Major thrombi } \\
\hline Negative & 37 & 11 & \\
\hline Positive & 4 & 0 & 0.567 \\
\hline \multicolumn{4}{|c|}{ Microvascular thrombi } \\
\hline Negative & 35 & 9 & \\
\hline Positive & 6 & 2 & 0.856 \\
\hline \multicolumn{4}{|l|}{ HCC stage } \\
\hline Early stage $^{a}$ & 30 & 5 & \\
\hline Advanced stage ${ }^{b}$ & 11 & 6 & 0.145 \\
\hline \multicolumn{4}{|l|}{ ICC stage } \\
\hline Early stage & 32 & 7 & \\
\hline Advanced stage & 9 & 4 & 0.435 \\
\hline
\end{tabular}

${ }^{a}$ Stage I and II; ${ }^{b}$ stage III and IV. AFP, $\alpha$-fetoprotein; CA19-9, carbohydrate antigen 19-9; GGT, $\gamma$-glutamyl transpeptidase; HbsAg, hepatitis B surface antigen; HCC, hepatocellular carcinoma; ICC, intrahepatic cholangiocarcinoma.

determinant of patient prognosis, was evaluated. As presented in Fig. 1A, the NLR value was compared between the early-stage group (HCC stage I and II) and the advanced-stage group (HCC stage III and IV), according to the TNM staging system. The NLR value was significantly higher in the advanced-stage group compared with that in the early-stage group $(3.19 \pm 2.34$ vs. $2.00 \pm 1.17 ; \mathrm{P}=0.001)$. However, there was no difference in NLR value between the ICC early-stage group and the ICC advanced-stage group according to TNM staging system (2.29 \pm 1.87 vs. $1.70 \pm 1.08 ; \mathrm{P}=0.301$; Fig. 1B).

Overall survival according to NLR. The OS rates of the patients in the advanced-stage group were significantly poorer compared with those in the early-stage group, according to the HCC staging system (1-year OS: 74.7 vs. 88.6\%; 2-year OS: 40.7 vs. 71.4\%; $\mathrm{P}=0.009$; Fig. 2). However, the difference in OS rates between the early-stage and advanced-stage patients in the ICC stage system were not significant (1-year OS: 87.7 vs. 66.2\%; 2-year OS: 73.8 vs. 46.2\%; $\mathrm{P}=0.169$; Fig. 3).

The OS rates of the patients with NLR $\geq 2.75$ were significantly lower compared with those of the patients with NLR $\leq 2.75$ (1-year OS: 63.6 vs. 89.9\%; 2-year OS: 24.2 vs. $72.1 \% ; \mathrm{P}=0.004)$. The median survival time was longer in the NLR $\leq 2.75$ group compared with that in the NLR $>2.75$ group (83.6 vs. 15 months; $\mathrm{P}=0.004$ ) (Fig. 4).

Prognostic significance of NLR in patients with $\mathrm{cHCC}-\mathrm{CC}$. The clinicopathological parameters were included in the univariate and multivariate analyses to further investigate the prognostic factors of patients with $\mathrm{cHCC}-\mathrm{CC}$. As presented in Table II, tumor size, HCC TNM stage and NLR were significant factors associated with OS in the univariate analysis. The significant predictors were then utilized for multivariate proportional hazard regression analysis. The multiple analysis results revealed that advanced stage in the HCC TNM staging system [hazard ratio (HR), 2.527; 95\% CI, 1.088-5.872; $\mathrm{P}=0.031]$ and NLR>2.75 (HR, 2.990; 95\% CI, 1.198-7.462; $\mathrm{P}=0.019)$ were independent prognostic factors of poor OS in cHCC-CC (Table II).

\section{Discussion}

cHCC-CC is a mixed carcinoma that is composed of two distinct tumor elements in which HCC and CC intimately coexist (4). The present study confirmed the prognostic value of NLR in a cohort of patients with cHCC-CC.

Inflammatory processes have been identified to serve a role in tumor progression (9). Growth and survival factors released from inflammatory cells can stimulate tumor formation, progression, angiogenesis, invasion and metastasis (23-25). The paradoxical roles of adaptive (lymphocyte immune cells) and innate leukocytes (circulating neutrophils) in inflammatory processes act as crucial opposing regulators in cancer occurrence (26). Immune cell-like neutrophils have been associated with increased angiogenesis and/or a poor prognosis, which is in part explained by the upregulation of cyclooxygenase-2 or the suppression of an antitumor adaptive immune response (27-29). However, lymphocytes have been essential components in tumor defense via killing tumor cells and inhibiting cell proliferation or migration $(9,30)$. Additionally, certain previous studies suggested that adaptive immune cells, 
A

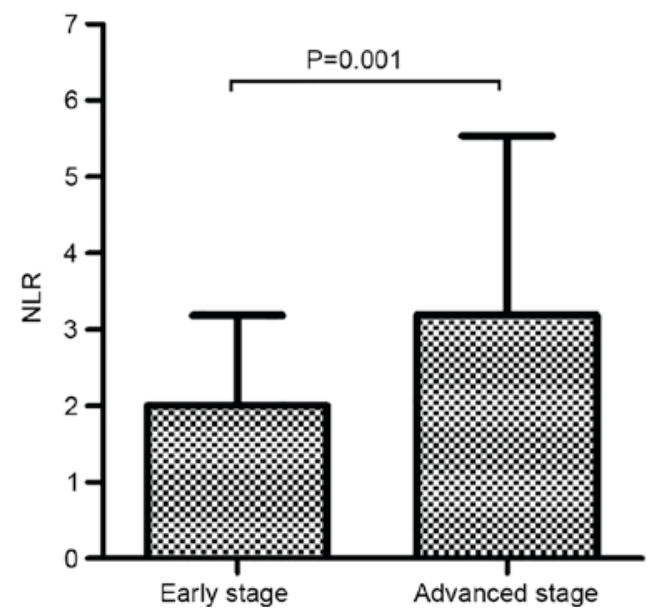

B

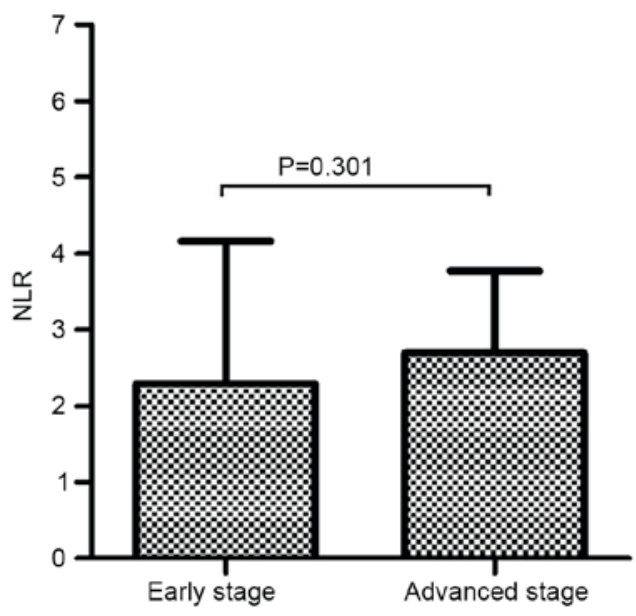

Figure 1. Association between NLR and the TNM staging system. (A) The level of NLR tended to increase with progressing tumor stage according to the hepatocellular carcinoma TNM staging system $(\mathrm{P}=0.001)$. (B) There was no significant difference in NLR between the early and advanced stages, according to the intrahepatic cholangiocarcinoma TNM staging system $(\mathrm{P}=0.301)$. NLR, neutrophil-to-lymphocyte ratio; TNM, Tumor-Node-Metastasis.

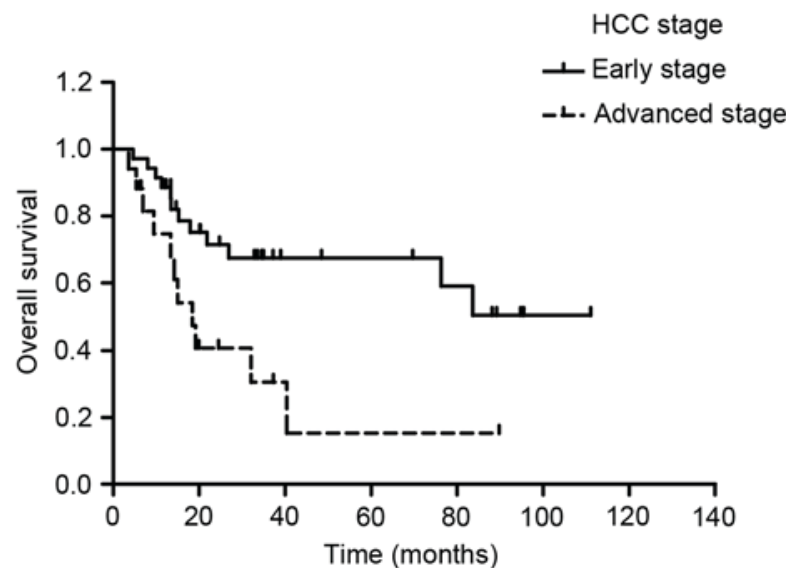

Figure 2. Kaplan-Meier curves revealing overall survival in patients who underwent surgical resection according to HCC Tumor-Node-Metastasis stage at diagnosis. Patients in the advanced-stage group exhibited significantly poorer overall survival rates compared with those in the early-stage group according to the HCC staging system $(\mathrm{P}=0.009)$. HCC, hepatocellular carcinoma.

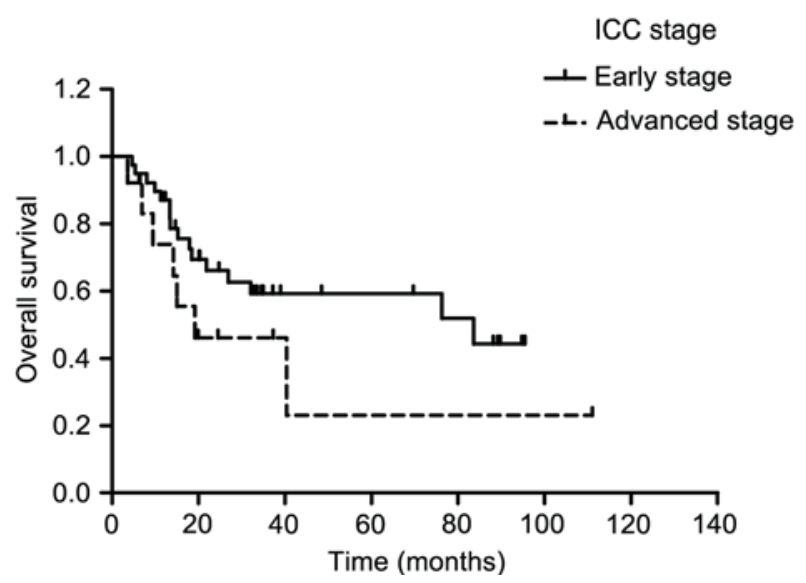

Figure 3. Kaplan-Meier curves revealing overall survival in patients who underwent surgical resection according to the ICC Tumor-Node-Metastasis stage at diagnosis. The difference between the early- and advanced-stage groups was not significant $(\mathrm{P}=0.169)$. ICC, intrahepatic cholangiocarcinoma.

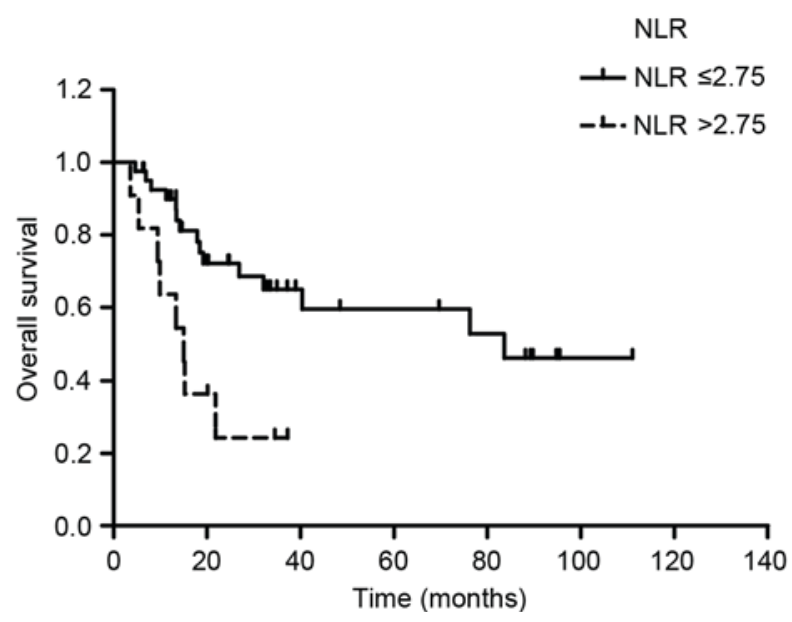

Figure 4. Kaplan-Meier curves revealing overall survival in patients according to NLR value at diagnosis. Patients with NLR $\geq 2.75$ exhibited significantly poorer overall survival rates compared with those with NLR $\leq 2.75(\mathrm{P}=0.004)$. NLR, neutrophil-to-lymphocyte ratio.

including B-lymphocytes, cluster of differentiation (CD) $8^{+}$ cytotoxic T-lymphocytes and $\mathrm{CD}^{+}$helper T-lymphocytes, served important roles in the modulation of cancer development via the lysis of tumor cells $(26,31)$.

Inflammation is a complex process that may be reflected by NLR, a practical biomarker. The present study demonstrated a substantial difference in NLR between early- and advanced-stage $\mathrm{HCC}(\mathrm{P}=0.001)$; however, there were no significant differences in NLR values between early and advanced ICC stage according to the TNM staging system $(\mathrm{P}=0.301)$. cHCC-CC has been included in the ICC section of the TNM staging system in accordance with the AJCC manual (21); however, a study with a larger cohort suggested that the HCC TNM staging system provided an better prognostic stratification for patients with cHCC-CC (6). Similarly, in the present study, the OS rates of patients with advanced HCC stage were significantly lower compared with those of patients with early stage disease, according to the TNM staging system. However, 
Table II. Univariate and multivariate analyses of factors associated with overall survival in 52 patients with combined hepatocellular-cholangiocarcinoma.

\begin{tabular}{|c|c|c|c|c|c|c|}
\hline \multirow[b]{2}{*}{ Characteristics } & \multirow[b]{2}{*}{$\mathrm{n}$} & \multirow[b]{2}{*}{$\%$} & \multicolumn{2}{|l|}{ Univariate } & \multicolumn{2}{|c|}{ Multivariate } \\
\hline & & & $\mathrm{HR}(95 \% \mathrm{CI})$ & P-value & HR (95\% CI) & P-value \\
\hline \multicolumn{7}{|l|}{ Age, years } \\
\hline$\leq 60$ & 44 & 84.6 & Reference value & & & \\
\hline$>60$ & 8 & 15.4 & $0.716(0.212-2.417)$ & 0.590 & & \\
\hline \multicolumn{7}{|l|}{ Gender } \\
\hline Female & 15 & 28.8 & Reference value & & & \\
\hline Male & 37 & 71.2 & $1.487(0.551-4.015)$ & 0.434 & & \\
\hline \multicolumn{7}{|l|}{ AFP, $n g / m l$} \\
\hline$\leq 25$ & 20 & 38.5 & Reference value & & & \\
\hline$>25$ & 32 & 61.5 & $1.296(0.546-3.077)$ & 0.556 & & \\
\hline \multicolumn{7}{|l|}{$\mathrm{CA} 19-9, \mathrm{U} / \mathrm{ml}$} \\
\hline$\leq 35$ & 36 & 69.2 & Reference value & & & \\
\hline$>35$ & 16 & 30.8 & $1.127(0.477-2.664)$ & 0.785 & & \\
\hline \multicolumn{7}{|l|}{ GGT, U/1 } \\
\hline$\leq 50$ & 23 & 44.2 & Reference value & & & \\
\hline$>50$ & 29 & 55.8 & $1.788(0.756-4.229)$ & 0.186 & & \\
\hline \multicolumn{7}{|l|}{ HbsAg } \\
\hline Negative & 6 & 11.5 & Reference value & & & \\
\hline Positive & 46 & 88.5 & $3.510(0.470-26.239)$ & 0.221 & & \\
\hline \multicolumn{7}{|l|}{ Tumor size, cm } \\
\hline$<5$ & 26 & 50.0 & Reference value & & & \\
\hline$\geq 5$ & 26 & 50.0 & $3.475(1.411-8.562)$ & 0.007 & $1.113(0.959-1.291)$ & 0.159 \\
\hline \multicolumn{7}{|l|}{ Tumor number } \\
\hline Solitary & 14 & 26.9 & Reference value & & & \\
\hline Multiple & 38 & 73.1 & $0.955(0.375-2.429)$ & 0.923 & & \\
\hline \multicolumn{7}{|c|}{ Lymph node metastasis } \\
\hline Negative & 45 & 26.5 & Reference value & & & \\
\hline Positive & 7 & 13.5 & $3.036(0.992-9.292)$ & 0.052 & & \\
\hline \multicolumn{7}{|l|}{ Major thrombus } \\
\hline Negative & 48 & 92.3 & Reference value & & & \\
\hline Positive & 4 & 7.7 & $3.121(0.912-10.684)$ & 0.070 & & \\
\hline \multicolumn{7}{|c|}{ Microvascular thrombus } \\
\hline Negative & 44 & 84.6 & Reference value & & & \\
\hline Positive & 8 & 15.4 & $1.676(0.556-5.054)$ & 0.305 & & \\
\hline \multicolumn{7}{|l|}{ HCC stage } \\
\hline Early stage $^{\mathrm{a}}$ & 35 & 67.3 & Reference value & & & \\
\hline Advanced stage $^{\mathrm{b}}$ & 17 & 32.7 & $2.882(1.258-6.607)$ & 0.012 & $2.527(1.088-5.872)$ & 0.031 \\
\hline \multicolumn{7}{|l|}{ ICC stage } \\
\hline Early stage & 39 & 75.0 & Reference value & & & \\
\hline Advanced stage & 13 & 25.0 & $1.853(0.758-4.528)$ & 0.176 & & \\
\hline \multicolumn{7}{|l|}{ NLR } \\
\hline$\leq 2.75$ & 41 & 78.8 & Reference value & & & \\
\hline$>2.75$ & 11 & 21.2 & $3.474(1.409-8.563)$ & 0.007 & $2.990(1.198-7.462)$ & 0.019 \\
\hline
\end{tabular}

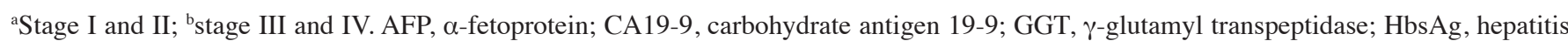
B surface antigen; HCC, hepatocellular carcinoma; ICC, intrahepatic cholangiocarcinoma; HR, hazard ratio; CI, confidence interval; NLR, neutrophil-to-lymphocyte ratio; HR, hazard ratio. 
there were no differences in OS between the early and advanced ICC stages, according to the TNM staging system $(\mathrm{P}=0.301)$. Furthermore, a recent study revealed that the NLR value exhibited a linear association with cancer progression staging (17). This may partly explain the difference in NLR value between early- and advanced-stage HCC present in the TNM staging system but not the ICC TNM staging system in the present study.

Increasing evidence has suggested that NLR is a prognostic factor for OS in various types of cancer. Various cut-off values have been used to describe the association between NLR and survival in these cancer types $(32,33)$. In the present study, a time-dependent ROC was performed to determine the optimal cut-off points for NLR. According to this, the present study demonstrated that patients could be divided into two groups (NLR $>2.75$ and NLR $\leq 2.75)$. Upon univariate and multivariate analyses, the present study confirmed that NLR was an independent prognostic factor. In contrast with the present study, a previous study revealed that an NLR value of $>2$ did not independently predict poorer overall survival in patients with cHCC-CC (34). This discrepancy may be due to differences in the assays using NLR. The previous study selected the median value as a cut-off point, whereas the present study used a time-dependent ROC curve to select a high-sensitivity value cut-off point for NLR.

There are certain limitations to the present study. Firstly, the total number of recruited patients was relatively small. Secondly, it was a retrospective study and was thus susceptible to bias in data selection and analysis. Other inflammatory markers, including C-reactive protein or procalcitonin, which have been demonstrated to be independent prognostic factors in patients with HCC (17) and ICC (35), are not routinely evaluated at Sun Yat-sen University. Finally, the present study did not evaluate NLR following surgery, and further investigation is required to determine whether this was associated with OS.

Despite these limitations, the present study suggested that pre-treatment NLR may be associated with OS in patients diagnosed with cHCC-CC and that analysis of the NLR should be introduced in clinical practice. Further prospective studies are required to evaluate cut-off values and confirm the results of the present study.

\section{Acknowledgements}

The present study was supported by National Natural Science Foundation of China (grant no. 81171890) and the Major National Scientific Research Projects of China (grant no. 2013CB910304).

\section{Competing interests}

The authors declare that they have no competing interests.

\section{References}

1. Allen RA and Lisa JR: Combined liver cell and bile duct carcinoma. Am J Pathol 25: 647-655, 1949.

2. Jarnagin WR, Weber S, Tickoo SK, Koea JB, Obiekwe S, Fong Y, DeMatteo RP, Blumgart LH and Klimstra D: Combined hepatocellular and cholangiocarcinoma: Demographic, clinical, and prognostic factors. Cancer 94: 2040-2046, 2002 .
3. Panjala C, Senecal DL, Bridges MD, Kim GP, Nakhleh RE, Nguyen JH and Harnois DM: The diagnostic conundrum and liver transplantation outcome for combined hepatocellularcholangiocarcinoma. Am J Transplant 10: 1263-1267, 2010.

4. Bosman FT, Carneiro F, Hruban RH and Theise ND: WHO classification of tumours of the digestive system. 4th Edition, International Agency for Research on Cancer Publisher, Lyon. World Health Organization, 2010.

5. Kim SH, Park YN, Lim JH, Choi GH, Choi JS and Kim KS: Characteristics of combined hepatocelluar-cholangiocarcinoma and comparison with intrahepatic cholangiocarcinoma. Eur J Surg Oncol 40: 976-981, 2014.

6. Chu KJ,Lu CD, Dong H, Fu XH,Zhang HW and Yao XP: Hepatitis $\mathrm{B}$ virus-related combined hepatocellular-cholangiocarcinoma: Clinicopathological and prognostic analysis of 390 cases. Eur J Gastroenterol Hepatol 26: 192-199, 2014.

7. Zheng W, Yao M, Sai W, Qian Q, Pan L, Qiu L, Huang J, Wu W and Yao D: Diagnostic and prognostic significance of secretory clusterin expression in patients with hepatocellular carcinoma. Tumour Biol 37: 999-1008, 2016.

8. Kassahun WT and Hauss J: Management of combined hepatocellular and cholangiocarcinoma. Int J Clin Pract 62: 1271-1278, 2008.

9. Coussens LM and Werb Z: Inflammation and cancer. Nature 420: 860-867, 2002.

10. Zahorec R: Ratio of neutrophil to lymphocyte counts-rapid and simple parameter of systemic inflammation and stress in critically ill. Bratisl Lek Listy 102: 5-14, 2001 (In English, Slovak).

11. Sarraf KM, Belcher E, Raevsky E, Nicholson AG, Goldstraw P and Lim E: Neutrophil/lymphocyte ratio and its association with survival after complete resection in non-small cell lung cancer. J Thorac Cardiovasc Surg 137: 425-428, 2009.

12. Yamanaka T, Matsumoto S, Teramukai S, Ishiwata R, Nagai Y and Fukushima M: The baseline ratio of neutrophils to lymphocytes is associated with patient prognosis in advanced gastric cancer. Oncology 73: 215-220, 2007.

13. Cho H, Hur HW, Kim SW, Kim SH, Kim JH, Kim YT and Lee K: Pre-treatment neutrophil to lymphocyte ratio is elevated in epithelial ovarian cancer and predicts survival after treatment. Cancer Immunol Immunother 58: 15-23, 2009.

14. An X, Ding PR, Li YH, Wang FH, Shi YX, Wang ZQ, He YJ, $\mathrm{Xu}$ RH and Jiang WQ: Elevated neutrophil to lymphocyte ratio predicts survival in advanced pancreatic cancer. Biomarkers 15: 516-522, 2010.

15. Gomez D, Farid S, Malik HZ, Young AL, Toogood GJ, Lodge JP and Prasad KR: Preoperative neutrophil-to-lymphocyte ratio as a prognostic predictor after curative resection for hepatocellular carcinoma. World J Surg 32: 1757-1762, 2008.

16. Kayadibi H, Sertoglu E, Uyanik M and Tapan S: Neutrophil-lymphocyte ratio is useful for the prognosis of patients with hepatocellular carcinoma. World J Gastroenterol 20: 9631-9632, 2014.

17. Oh BS, Jang JW, Kwon JH, You CR, Chung KW, Kay CS, Jung HS and Lee S: Prognostic value of C-reactive protein and neutrophil-to-lymphocyte ratio in patients with hepatocellular carcinoma. BMC Cancer 13: 78, 2013.

18. Xiao WK, Chen D, Li SQ, Fu SJ, Peng BG and Liang LJ: Prognostic significance of neutrophil-lymphocyte ratio in hepatocellular carcinoma: A meta-analysis. BMC Cancer 14: $117,2014$.

19. Shen SL, Fu SJ, Huang XQ, Chen B, Kuang M, Li SQ, Hua YP, Liang LJ and Peng BG: Elevated preoperative peripheral blood monocyte count predicts poor prognosis for hepatocellular carcinoma after curative resection. BMC Cancer 14: 744, 2014.

20. Chen Q, Yang LX, Li XD, Yin D, Shi SM, Chen EB, Yu L, Zhou ZJ, Zhou SL, Shi YH, et al: The elevated preoperative neutrophil-to-lymphocyte ratio predicts poor prognosis in intrahepatic cholangiocarcinoma patients undergoing hepatectomy. Tumour Biol 36: 5283-5289, 2015.

21. Edge S, Byrd D, Compton C, Fritz A, Greene F and Trotti A: AJCC cancer staging manual 7. American Joint Committee on Cancer, 2010

22. Heagerty PJ, Lumley T and Pepe MS: Time-dependent ROC curves for censored survival data and a diagnostic marker. Biometrics 56: 337-344, 2000.

23. Jaiswal M, LaRusso NF, Burgart LJ and Gores GJ: Inflammatory cytokines induce DNA damage and inhibit DNA repair in cholangiocarcinoma cells by a nitric oxide-dependent mechanism. Cancer Res 60: 184-190, 2000. 
24. de Visser KE, Eichten A and Coussens LM: Paradoxical roles of the immune system during cancer development. Nat Rev Cancer 6: 24-37, 2006.

25. Jackson JR, Seed MP, Kircher CH, Willoughby DA and Winkler JD: The codependence of angiogenesis and chronic inflammation. FASEB J 11: 457-465, 1997.

26. Ishigami $S$, Natsugoe $S$, Tokuda $K$, Nakajo $A$, Che $X$, Iwashige $H$, Aridome K, Hokita S and Aikou T: Prognostic value of intratumoral natural killer cells in gastric carcinoma. Cancer 88: 577-583, 2000.

27. Liu CH, Chang SH, Narko K, Trifan OC, Wu MT, Smith E, Haudenschild C, Lane TF and Hla T: Overexpression of cyclooxygenase- 2 is sufficient to induce tumorigenesis in transgenic mice. J Biol Chem 276: 18563-18569, 2001.

28. Schoppmann SF, Birner P, Stöckl J, Kalt R, Ullrich R, Caucig C, Kriehuber E, Nagy K, Alitalo K and Kerjaschki D: Tumor-associated macrophages express lymphatic endothelial growth factors and are related to peritumoral lymphangiogenesis. Am J Pathol 161: 947-956, 2002.

29. Dannenberg AJ and Subbaramaiah K: Targeting cyclooxygenase-2 in human neoplasia: Rationale and promise. Cancer Cell 4: 431-436, 2003.

30. Mantovani A, Allavena P, Sica A and Balkwill F: Cancer-related inflammation. Nature 454: 436-444, 2008.

31. Zou W: Immunosuppressive networks in the tumour environment and their therapeutic relevance. Nat Rev Cancer 5: 263-274 2005 .
32. Perez DR, Baser RE, Cavnar MJ, Balachandran VP, Antonescu CR, Tap WD, Strong VE, Brennan MF, Coit DG, Singer S and Dematteo RP: Blood neutrophil-to-lymphocyte ratio is prognostic in gastrointestinal stromal tumor. Ann Surg Oncol 20: 593-599, 2013.

33. Williams KA, Labidi-Galy SI, Terry KL, Vitonis AF, Welch WR, Goodman A and Cramer DW: Prognostic significance and predictors of the neutrophil-to-lymphocyte ratio in ovarian cancer. Gynecol Oncol 132: 542-550, 2014.

34. Cai X, Zhai J, Kaplan DE, Zhang Y, Zhou L, Chen X, Qian G, Zhao Q, Li Y, Gao L, et al: Background progenitor activation is associated with recurrence after hepatectomy of combined hepatocellular-cholangiocarcinoma. Hepatology 56: 1804-1816, 2012.

35. Zeng $\mathrm{X}$ and Tao H: Diagnostic and prognostic serum marker of cholangiocarcinoma (Review). Oncol Lett 9: 3-8, 2015.

This work is licensed under a Creative Commons Attribution-NonCommercial-NoDerivatives 4.0 International (CC BY-NC-ND 4.0) License. 